\title{
DIAGNÓSTICO E TRATAMENTO DA OSTEOPOROSE
}

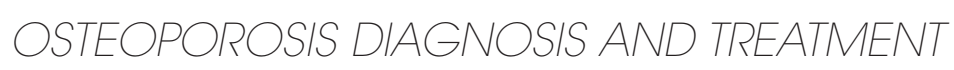

Márcio Passini Gonçalves de Souza

\section{RESUMO}

Um artigo de atualização do conhecimento sobre osteoporose corre o risco de ficar desatualizado precocemente, devido ao grande interesse que o estudo e a pesquisa sobre OP despertam hoje nos pesquisadores, nas indústrias farmacêuticas e de equipamentos, nos governos, e até na OMS. Todo ortopedista conhece a OP pelo seu efeito mais deletério, a fratura osteoporótica (FxOP). Por ser uma patologia de quadro clínico não específico a OP sem fratura não levanta suspeita. A FxOP tem um custo econômico (pelo tratamento), social (por suas sequelas) e médico (por óbitos). Muitas fraturas poderiam ser evitadas pelo diagnóstico da OP antes da primeira fratura e, então, muitas incapacidades temporárias e definitivas poderiam ser evitadas, muitas vidas poderiam ser salvas. O conhecimento dos fatores de risco para osteoporose desperta a suspeita e a densitometria óssea ajuda no diagnóstico. O tratamento deve ter por base a fisiopatologia da doença. Assim, na prevenção ou no tratamento da OP, devemos diminuir a atividade do osteoclasto ou aumentar a atividade do osteoblasto, ou os dois. O tratamento ideal é aquele que diminui a incidência de fraturas por melhorar a geometria do osso e sua microarquitetura. $\mathrm{O}$ tecido ósseo recém-formado deve ter boa qualidade celular e de matriz, mineralização normal com boa proporção entre osso mineralizado (resistente mecanicamente) e não mineralizado (flexível) e sem acúmulo de danos. O tratamento ideal deve ter taxa de remodelação positiva e efeito terapêutico rápido e duradouro. Este efeito deve ser facilmente detectável. Deve ser seguro.

Descritores - Osteoporose/fisiopatologia; Osteoporose/diagnóstico; Osteoporose/prevenção \& controle; Fraturas ósseas

\section{ABSTRACT}

An article regarding the latest understanding of osteoporosis $(O P)$ runs the risk of quickly becoming obsolete due to the fact that research and studies about OP today are generating a great amount of interest in researchers, the pharmaceutical and medical equipment industries, governments, and even the WHO. Every orthopedist knows OP by its most deleterious effect, the osteoporotic fracture (FxOP). OP without a fracture does not arouse suspicion because it is a pathology with a nonspecific clinical profile. The FxOP has an economic cost (for treatment), a social cost (for its sequelae), and a medical cost (for deaths). Many fractures could be avoided by a diagnosis of OP prior to the first fracture and, therefore, many temporary and permanent disabilities could be avoided and many lives saved. Awareness of the risk factors for osteoporosis raises suspicion and bone densitometry aids diagnosis. Treatment should be based on the physiopathology of the disease. Likewise, in prevention or in treatment of OP, we should reduce the activity of osteoclasts or increase the activity of osteoblasts, or both. Treatment that reduces the incidence of fractures by improving bone's geometry and microarchitecture is ideal. Newly formed bone tissue must have good cellular and matrix quality as well as normal mineralization, a good ratio of mineralized (mechanically resistant) bone to nonmineralized (flexible) bone, and no accumulated damage. The ideal treatment should have a positive rate of remodeling and fast and lasting therapeutic effects. This effect must be easily detectable. It must be safe.

Keywords - Osteoporosis/physiopathology; Osteoporosis/diagnosis; Osteoporosis/prevention \& control

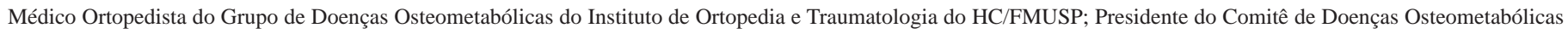
e Degenerativas da SBOT e Presidente da Associação Brasileira Ortopédica de Osteometabolismo (ABOOM).

Correspondência: Rua Pernambuco, 88 - 14º andar - 01240-020 - São Paulo, SP - E-mail: marciopassini@terra.com.br 


\section{INTRODUÇÃO}

Durante muitos anos, o estudo da osteoporose, pela pouca praticidade do seu conhecimento, foi relegado a um plano secundário. Hoje, além de ser um assunto muito pesquisado em todo o mundo, seu conhecimento é objetivo e útil. Um artigo de atualização sobre o tema torna-se rapidamente desatualizado pelo fato de que os conhecimentos sobre o assunto evoluem diariamente.

Os conhecimentos básicos sobre osteoporose (OP) estão entranhados na consciência dos ortopedistas desde o início do século XX. A palavra osteoporose surgiu do estudo histológico de um osso osteoporótico por Jean Georges Chretien Frederic Martin Lobstein, patologista francês, em 1830 apud Oliveira ${ }^{(1)}$, mas popularizou-se entre os ortopedistas como um sinal radiológico, que significava rarefação óssea, em fraturas causadas por traumas de baixa energia. Este mesmo sinal os radiologistas chamam osteopenia.

No final do século passado o conceito de osteoporose mudou progressivamente da definição de uma doença bem específica, feita por Albright em 1941, para o conceito atual de uma desordem esquelética, o que engloba muitas patologias, nas quais a microarquitetura do tecido ósseo está deteriorada ${ }^{(2,3)}$. Tanto o osso cortical como o esponjoso são afetados. Também a macroarquitetura dos ossos pode estar modificada. A densidade mineral óssea (DMO) está diminuída. Isto leva a um comprometimento da resistência do osso a traumas de baixa energia ${ }^{(4)}$. O osso fica frágil, predisposto a um aumento da ocorrência de fraturas. É a alta incidência destas fraturas, chamadas de fraturas osteoporóticas (FxOPs) que traz importância ao estudo da osteoporose.

A osteoporose participa não só para aumentar a frequência das fraturas, mas também para aumentar as possibilidades de formatos diferentes, desde fraturas sem manifestação clínica, como as chamadas fraturas morfométricas do corpo vertebral, passando por fraturas incompletas, até fraturas cominutivas muito instáveis que apresentam impossibilidade técnica de remontagem anatômica do osso. Algumas fraturas podem não ser detectáveis; outras, como as do corpo vertebral, podem deixar sequelas muito dolorosas; e outras podem levar o paciente ao óbito ou à incapacidade física permanente, como as fraturas da extremidade proximal do fêmur.

O aumento absoluto e relativo da população idosa e os hábitos pouco saudáveis dos infantes e adolescentes estão levando a um aumento muito grande da incidência de OP e também das FxOPs.
São múltiplas as causas para o aparecimento e/ou desenvolvimento da OP. Chamamos de OP primária quando as causas são naturais (menopausa e senilidade). Falamos em OP secundária quando há uma causa primária (certos medicamentos, outras doenças, sedentarismo etc.). Quando as causas são desconhecidas chamamos de OP idiopática.

\section{DIAGNÓSTICO}

Diz-se que a OP sem fratura atual ou sem microfratura é uma doença silenciosa porque não tem sintomas específicos que possam levar-nos a suspeitá-la. Não parece ser verdade. Todas as doenças mediadas pelo osteoclasto são dolorosas. A OP talvez seja menos dolorosa, ou talvez a dor possa passar despercebida por ser mais branda. Muitas lombalgias e dorsalgias podem ser de origem osteoporótica e o ortopedista deve estar alertado para esta possibilidade. A OP também não tem sinais clínicos patognomônicos. O aumento da cifose torácica e a perda de estatura talvez sejam os sinais mais suspeitos.

Por sua natureza multifatorial, seu caráter sindrômico e suas baixas manifestações clínicas, a OP é difícil de diagnosticar. Na maior parte das vezes a OP é diagnosticada pelos ortopedistas pela sua consequência mais deletéria, a fratura osteoporótica.

Devemos, então, estarmos atentos para o diagnóstico do risco de uma pessoa ter OP. A tentativa de diagnosticar e tratar precocemente a OP, antes da ocorrência da primeira fratura, levou ao estudo dos fatores de risco para $\mathrm{OP}^{(5)}$.

\section{Fatores de risco para osteoporose}

É preciso distinguir entre fatores de risco para osteoporose e fatores de risco para fraturas osteoporóticas. Nos primeiros estuda-se a possibilidade de o paciente apresentar osteoporose e a necessidade da realização de exames subsidiários para comprová-la. Nos fatores de risco para fratura osteoporótica estuda-se a possibilidade do paciente vir a sofrer uma fratura por fragilidade óssea e, então, a existência de osteoporose é um dos fatores de risco.

Os fatores de risco mais valorizados para osteoporose são: o gênero feminino, as etnias amarela e branca, a idade mais avançada, a precocidade do início da menopausa, a hereditariedade (presença de osteoporose ou de fratura osteoporótica entre os ancestrais e os colaterais), história pregressa de fraturas osteoporóticas, erros nutricionais (baixa ingestão de cálcio, baixa ingestão 
de vitamina $\mathrm{D}_{3}$ ou baixa insolação para produção da mesma, situações para má absorção de alimentos etc.), maus hábitos (ingestão exagerada de café, álcool, tabaco), sedentarismo, certas medicações (glicocorticoides, anticonvulsivantes) e doenças como a artrite reumatoide e quase todas as doenças inflamatórias sistêmicas.

Apesar de os fatores de risco para osteoporose serem bastante conhecidos há muito tempo, ainda não há uma fórmula numérica científica para avaliá-los separadamente e no contexto geral. E talvez nem venha a existir. Dependendo da população estudada estes fatores de risco têm valores relativos diferentes.

O desenvolvimento do densitômetro veio ajudar no diagnóstico, mas surgem então as perguntas: Quando realizar uma densitometria? Quando repetir a avaliação? E novamente é necessário avaliar os fatores de risco para a osteoporose.

Tabela 1 - Valor relativo dos fatores de risco para osteoporose

\begin{tabular}{c|c|c|c|c|c}
\hline Coeficiente & Valor 1 & $\mathbf{2}$ vezes & $\mathbf{4}$ vezes & $\mathbf{8}$ vezes & Absoluto \\
\hline Gênero & Masculino & & Feminino & & \\
\hline Etnia & Negra & Parda & Branca & Amarela & \\
\hline Idade & 20 & 50 & 60 & 70 & 80 \\
\hline IMC & $>30$ & 27 a 30 & 24 a 27 & 20 a 24 & \\
\hline Idade menop. & $>52$ & 48 a 52 & 44 a 48 & Até 44 & \\
\hline FxOP Prévia & & & QQ FXOP & Vért. & Fêmur \\
\hline FxOP Pais & & Outras & Vért. & Fêmur & \\
\hline Hábitos & Tabaco & Álcool & Café & & \\
\hline Ativ. física & Diária & Frequente & Ocasional & Sedentária & \\
\hline ISDA & & & Anticonv. & Artr. reum. & Corticoter. \\
\hline
\end{tabular}

Na Tabela 1 estão distribuídos os fatores de risco para OP e seu valor, relativamente aos demais, conforme publicada em várias fontes de informação. A coluna "Valor 1" é a base para os cálculos. Assim, o gênero feminino tem quatro vezes mais chance de ter OP em relação ao masculino, a etnia amarela tem duas vezes mais que a branca $(8 / 4=2)$ e esta quatro vezes mais que a negra $(4 / 1=4)$. A consideração de vários fatores leva à solicitação de uma avaliação densitométrica. Somar a “pontuação” não traz resultado prático. Por exemplo, na coluna da direita a existência de qualquer dos fatores exige a realização de densitometria óssea. A experiência clínica, nesta e em qualquer outra patologia, leva o médico a desconfiar da existência da patologia e a procurar o diagnóstico. No caso da OP, a desconfiança surge da existência dos fatores de risco.

\section{Densitometria}

O densitômetro é um aparelho gerador de duplo feixe de raios-X que atravessa uma região do corpo do paciente. Um colimador colhe a radiação emitida, avaliando a quantidade de cálcio pela área medida. Um computador analisa os resultados obtidos e os compara com um banco de dados de pessoas da mesma etnia, peso, altura e idades de 20 até 100 anos. Os resultados são apresentados em gramas $/ \mathrm{cm}^{2}$ e comparados à média das pessoas de 20 anos de idade (T score), que representa o valor do pico da massa óssea. Também são comparados aos valores médios da DMO das pessoas de mesma idade (Z score). São calculadas as porcentagens relativas e os desvios padrões (DPs) das médias. Os resultados são considerados, conforme consenso da OMS, como Normal, quando a densitometria mostra até -1 desvio padrão no $T$ score; Osteopenia, de -1 a $-2,5$ DPs e Osteoporose de -2,5 DPs para mais. Há ainda a denominação de Osteoporose estabelecida quando, além do DP $<-2,5$, o paciente apresenta uma fratura osteoporótica. Hoje se considera como portador de OP qualquer paciente que tenha tido uma FxOP. O Z score com valores iguais ou menores que -2 é sugestivo de uma possível OP secundária.

Como qualquer exame subsidiário, a densitometria deve ser realizada quando há indícios suficientes da possibilidade do paciente ser portador da patologia. A desconfiança é despertada pela existência de fatores de risco para osteoporose. Não havendo fatores de risco, a regra é realizar uma primeira avaliação densitométrica em todas as pessoas de mais de 65 anos de idade, e em todas as mulheres de 50 anos que tiveram menopausa precoce. $\mathrm{O}$ exame deve ser repetido de um a três anos, dependendo de critério clínico, ou para controle de tratamento.

\section{Fatores de risco para fraturas osteoporóticas}

Os fatores de risco para fraturas osteoporóticas são os mesmos fatores de risco para osteoporose, acrescidos do resultado de densitometria. São importantes também os fatores de risco para quedas, mas devemos lembrar que traumas comuns, de baixa energia, não provocam fraturas em pessoas hígidas. O próprio conceito de fratura osteoporótica é o de "fratura simples ou complexa que ocorre em pessoa portadora de osteoporose, aparente ou não aparente, provocada por um trauma de baixa energia”.

Não há relação segura entre a ocorrência de fraturas e o resultado da densitometria. A densitometria mede a massa óssea calcificada, mas não mede a qualidade 
desta massa óssea. Um caso conhecido é o do aumento da DMO densitométrica pelo uso do fluoreto de sódio, muito usado no passado no tratamento da osteoporose radiológica, que promovia maior fragilidade óssea. Outro exemplo é o do estrôncio, presente no ranelato de estrôncio, promissor meio de tratamento da osteoporose, que, por sua maior massa atômica e maior raio atômico, promove maior DMO densitométrica pela maior atenuação do feixe de raios-X do densitômetro.

Certamente os pacientes classificados como portadores de “osteoporose densitométrica” têm maior taxa de incidência de fraturas que os demais, e esta taxa é inversamente proporcional à DMO. Mas o número de fraturas osteoporóticas é muito maior entre os classificados como "osteopênicos densitométricos", e mesmo os "normais densitométricos" sofrem fraturas osteoporóticas em grande número. Isto ocorre porque as populações “normal” e "osteopênica” são maiores que a população osteoporótica ${ }^{(6)}$.

Um problema sério de saúde pública é então identificar a pessoa não portadora de OP densitométrica, porém suscetível de vir a ter uma fratura osteoporótica. Atualmente há um índice epidemiológico sendo estudado, sob o patrocínio pela OMS, o índice FRAX (Fracture Assessment Tool) $)^{(7)}$, que avalia estatisticamente os fatores de risco de uma pessoa, e os valoriza, e nos dará a chance, em porcentagem, da pessoa estudada vir a ter uma fratura osteoporótica em 10 anos $^{(8)}$. No Brasil já há estudos em andamento visando o estabelecimento do índice FRAX para a população brasileira.

Em estudos clínicos com pacientes tratados com bisfosfonatos orais e controlados com placebo, a perda de massa óssea densitométrica do grupo placebo é acompanhada de aumento da incidência de fraturas, e o ganho de massa óssea densitométrica até 5\% é acompanhado de diminuição proporcional desta incidência. Acima de 5\%, a diminuição da prevalência de fraturas se mantém, mas sem diminuição proporcional ao ganho de massa óssea densitométrica ${ }^{(9)}$.

\section{TRATAMENTO}

Estabelecido o diagnóstico de OP e estabelecido o risco de uma FxOP, deve-se decidir pelo tratamento profilático e/ou pelo curativo. A maior parte das intervenções serve aos dois objetivos. É óbvio que quando se faz a prevenção ou o tratamento da OP também está se fazendo a prevenção da FxOP.

Antes de discutirmos os tratamentos, vamos relembrar a REMODELAÇÃO ÓSSEA.
O osso é um tecido vivo que sofre constantemente um processo de troca de tecido antigo por tecido novo. O mediador deste processo é o osteócito. De tempos em tempos (mais ou menos mil dias) o osteócito entra em apoptose, isto é, a morte programada da célula. Na proximidade da apoptose ele produz sinalizadores para que células mesenquimais pluripotentes formem osteoblastos.

Estímulo semelhante ocorre quando o osso é submetido a esforços físicos para os quais não está preparado. Seja por pressões sobre as proteínas da membrana celular ${ }^{(10)}$, seja pela estimulação do cílio primário, organela do osteócito que detecta estas tensões, há sinalização às células mesenquimais para formar osteoblastos.

O osteoblasto produz o fator RANK (Receptor Activator of Nuclear factor Kappa beta) que sinaliza para as células hematopoiéticas formarem osteoclastos e também ativa a borda em escova destes osteoclastos.

Em 20 dias os osteoclastos reabsorvem parte do tecido ósseo, formando as lacunas de Howship. Agora os osteoblastos irão preencher estas lacunas com matriz proteica e finalmente nela depositarão cristais de hidroxiapatita. Este processo demora 180 dias para ser completado.

Havendo distúrbio deste remodelamento, pela maior ação proporcional do osteoclasto em relação ao osteoblasto, haverá uma pobre formação de tecido ósseo que, dependendo da gravidade, poderá ser osteopenia ou osteoporose.

Assim, na prevenção ou no tratamento da OP, devemos diminuir a atividade do osteoclasto ou aumentar a atividade do osteoblasto, ou os dois.

Parece ideal estimular a formação óssea estimulando a ação dos osteócitos ou dos osteoblastos, mas estes estimulam os osteoclastos, que agem em 20 dias, enquanto os osteoblastos irão gastar 180 dias para reparar a lacuna deixada pelo osteoclasto. Isto explica porque certos tratamentos anabólicos, isto é, que estimulam os osteoblastos, nem sempre alcançam os resultados esperados.

São tratamentos anabólicos: a atividade física, o calcitriol (vitamina D), a associação cálcio + calcitriol, esteroides anabolizantes, hormônio de crescimento, o paratormônio (PTH) e seu derivado, a teriparatida e o ranelato de estrôncio.

São tratamentos anticatabólicos, isto é, que inibem a ação do osteoclasto: a atividade física, a associação cálcio + calcitriol, os metabolitos ativos do calcitriol, as terapias de reposição de estrógeno (TRE) e de reposição hormonal (TRH), os SERMs (estimuladores seletivos dos receptores de estrógeno), os bisfosfonatos, a osteoprotegerina (OPG) e o ranelato de estrôncio. 


\section{Atividade física}

É o mais barato meio de prevenção e coadjuvante do tratamento. Os exercícios com peso e os exercícios de velocidade são os mais eficazes para o ganho de massa óssea. Além disso, o ganho de massa muscular e a melhoria da velocidade de resposta motora neuromuscular diminuem as quedas e o risco de fraturas nos pacientes. O efeito piezo elétrico da atividade física, ou a ação do cílio primário, estimulam os osteócitos, via osteoblastos, a promoverem a formação de osso novo.

Comparando idosos que praticam atividade física com idosos sedentários, há menores incidências de fraturas do quadril nos ativos ${ }^{(11)}$.

\section{Suplementação de cálcio}

O cálcio participa do cristal de hidroxiapatita $\left(\mathrm{Ca}_{10}\left(\mathrm{PO}_{4}\right)_{6}(\mathrm{OH})_{2}\right)$ que dá resistência mecânica ao osso. Na composição do tecido ósseo este cristal corresponde a $65 \%$. O cálcio atua também na coagulação sanguínea, na regulação metabólica por meio das metaloenzimas (alfa-amilase, fosfolipases, etc), na secreção de hormônios e de neurotransmissores e na aderência celular. Por sua presença na molécula de troponina, que regula a contratilidade da actina e miosina, o cálcio participa da contração muscular, inclusive do coração, e é a importância desta ação que faz com que, biologicamente, a calcemia permaneça o mais constante possível.

O cálcio existe na natureza em todos os seres vivos. As maiores fontes são o leite e os laticínios. Também são muito ricos: a sardinha, o feijão e os vegetais de folhas escuras. Nem sempre a ingestão de alimentos ricos em cálcio redunda na absorção deste pelo intestino. Esta absorção depende de o cálcio estar sob a forma de sais absorvíveis. Assim, a presença de ácido oxálico, vitamina C, fitatos (presentes na verdura cozida), certas fibras, proteínas e até mesmo lactose, podem formar compostos insolúveis ou não absorvíveis.

Outra fonte de cálcio é o exoesqueleto de moluscos. Daí tira-se o carbonato de cálcio, solúvel e absorvível em PH ácido. Por esta característica química, o carbonato de cálcio é pouco absorvível em pessoas idosas (devido à hipocloridria) e pacientes que tomam antiácidos etc. Nestas situações e nos casos de nefrolitíase usa-se o citrato de cálcio, mais absorvível e acidificante da urina. O fosfato tribásico de cálcio é usado nos casos de idosos com baixa ingestão de fósforo (raros), institucionalizados e com dificuldades de se alimentar.

No intestino, o cálcio é absorvido por via paracelular e por via transcelular. A via paracelular é passiva e depende da quantidade de cálcio no bolo alimentar, da velocidade de digestão, do $\mathrm{PH}$ do quilo e do sal de cálcio, além da presença de outros produtos já citados acima. A via transcelular é ativa e depende da presença da calbindina, sintetizada pela vitamina D.

Todo o cálcio presente no sangue é filtrado pelos glomérulos renais e a maior parte dele é reabsorvido pelos túbulos. Uma parte, de 100 a 300mg são eliminados diariamente pela urina e precisa ser reposta.

Nas pessoas com mais de 50 anos, fazendo ou não TRH, é imprescindível completar a dieta com suplementação diária de cálcio até $1.500 \mathrm{mg}$ em duas tomadas por dia. Uma dieta diária não láctea tem até 700mg e uma rica em laticínios tem até $950 \mathrm{mg}$. Oferece-se a mais para que o organismo aproveite o que necessita.

Os produtos farmacêuticos são denominados de acordo com a quantidade de cálcio elemento que eles contém no comprimido ou envelope e não pela quantidade do sal. Assim, 1.250mg de carbonato de cálcio aparecem com "cálcio 500".

A administração isolada de cálcio é eficiente para diminuir a incidência de fraturas ${ }^{(11)}$.

\section{Vitamina D}

A vitamina D é um "quase hormônio". Atua na absorção intestinal do cálcio alimentar e na reabsorção tubular renal do cálcio urinário. Reduz os níveis de PTH e estimula a osteogênese pelos osteoblastos. Tem ação antibiótica na árvore respiratória. Atua na modulação do equilíbrio no SNC. Facilita o aumento da força muscular principalmente na sarcopenia. Estimula a diferenciação e inibe a proliferação celular atuando então como protetor contra câncer de mama, próstata e intestino. Sua necessidade aumenta com a idade ${ }^{(12)}$. É produzida naturalmente por ação dos raios UVB do Sol sobre o 7-dihidrocolesterol circulante sob a pele irradiada, transformando-o no colicalciferol. Este, que já tem hidroxila em sua molécula, recebe outra hidroxila no carbono 25, ao passar pelo fígado, formando o calcidiol ou 25hidrocolicalciferol. A terceira hidroxila é fixada ao carbono 1 pela hidroxilase, no rim, formando o calcitriol ou 1,25-dihidrocolicalciferol. O colicalciferol ou vitamina D3 existe no fígado de peixes de águas frias, em ovos e em leites enriquecidos. Existe pouco no leite humano. Seu isômero, o ergosterol, ou vitamina D2, existe nos vegetais. As vitaminas D3, D2 e o calcidiol são inativos. O calcidiol é a forma de depósito. O calcitriol e seu metabolito alfacalcidol são as formas ativas na absorção do cálcio da luz do intestino e na reabsorção tubular 
renal do cálcio urinário. Eles têm vida muito curta e por isso não são dosados. O calcidiol é dosável e deve permanecer entre 32 a $100 \mathrm{ng} / \mathrm{mL}$ de soro ${ }^{(13)}$. Solicitar ao laboratório como "25-OH-Vitamina D” sérica. Para manter este nível o ideal é a ingestão de 800 a $1.200 U I$ de vitamina D3 por dia.

Há várias formulações no comércio. Quando associada ao cálcio, em geral há 200UI/comprimido. Existem outras preparações que associam o colicalciferol com o palmitato de retinol (vitamina A) e o alfatocoferol (vitamina E). Por exemplo, o Ad-til tem 250UI de Vitamina D e 1.250 UI de vitamina A por gota (40gotas $/ \mathrm{ml}$ ). Usa-se 40gotas por dia para repor a concentração ideal no soro (por cerca de três meses) e 40gotas por semana para a manutenção.

A associação de cálcio e vitamina D é eficiente na diminuição da incidência de fraturas ${ }^{(14)}$.

\section{Esteroides anabolizantes e hormônio de crescimento}

Atuam melhorando a formação da matriz proteica e na estimulação dos osteoblastos. Devido aos seus efeitos adversos são pouco utilizados. Na OP secundária por hipogonadismo masculino o uso de metil testosterona pelos urologistas é frequente e eficiente.

\section{Teriparatida e PTH}

O paratohormônio é formado por 84 aminoácidos dispostos em cadeia linear. A teriparatida é seu homólogo, só com os aminoácidos 1 ao 34, obtido pela técnica do DNA recombinante. Os dois, quando administrados de forma contínua, aumentam a ligação do RANK (RANKL) ao pré-osteoclasto estimulando a replicação do mesmo e ao osteoclasto estimulando sua ação de reabsorção de tecido ósseo. São então grandes reabsorvedores de osso (osteíte fibrosa cística). Porém, quando usados de forma diária, em pequenas doses, inibem o sistema RANKL e aumentam a OPG, inibindo então a reabsorção óssea. Neste caso, também estimulam a replicação e atividade do osteoblasto endostal e periostal. Com isto: aumentam a espessura da cortical, aumentam a secção transversa do osso e aumentam a espessura e conexão das trabéculas ${ }^{(15)}$. Isto dá mais resistência mecânica ao osso ${ }^{(16)}$. É usado sob a forma de microinjeções subcutâneas diárias por meio de uma "caneta” com 28 doses. Está muito indicada em pacientes com alto risco de fraturas e/ou refraturas ${ }^{(17)}$. Atualmente muitos estudos vêm sendo desenvolvidos procurando associar o uso da teriparatida concomitantemente ou sequencialmente com antirreabsortivos ${ }^{(18-20)}$.

\section{Terapia de reposição hormonal e estrogênica (TRH e TRE)}

São eficientes na prevenção da OP pós menopausa, mas não em seu tratamento. Devem ser iniciadas logo após a menopausa e controladas por ginecologista devido aos seus potenciais efeitos adversos. O maior problema é o aumento da ocorrência de câncer de mama, além dos distúrbios tromboembólicos.

\section{SERMs}

Os SERMs ou moduladores seletivos de receptores de estrógenos são usados quando as pacientes têm risco aumentado para câncer de mama. Eles inibem os receptores de estrógeno da mama e do útero, protegendo estes dois órgãos da ação deletéria do estrógeno. O mais usado é o citrato de tamoxifeno.

Para a prevenção e tratamento do OP, em substituição à TRH, foram desenvolvidos outros SERMs, com ação de estimulação estrogênica sobre os receptores de estrógeno do osso, aparelho cardiovascular e lipídeos. Assim, previnem e tratam a OP, previnem a hipercolesterolemia e as placas ateromatosas vasculares, e não estimulam o desenvolvimento de câncer de mama e útero. São eles o cloridrato de raloxifeno e o lasofoxifeno.

\section{Bisfosfonatos}

Bisfosfonatos (ou geminal bisfosfonatos) são polifosfatos que têm pelo menos uma conexão P-C-P (fósforo - carbono - fósforo) na molécula. Foram sintetizados pela primeira vez por Menschutkin em 1865 como anticorrosivos industriais. Mais tarde passou-se a utilizá-los como amaciantes de “águas duras” (águas alcalinas) em lavanderias e nas tubulações de águas (para impedir a deposição de carbonato de cálcio nos encanamentos).

Em 1968 Fleish e Russel descobriram o pirofosfato no plasma e na urina e, em 1970, descobriram que o pirofosfato inibe a precipitação do carbonato de cálcio nos vasos e vias urinárias sendo, portanto, nosso “amaciante” biológico. Pelo uso clínico que já se fazia, desde 1968, do etidronato em doenças osteometabólicas, passaram a investigar o uso dos bisfosfonatos em OP e doença óssea de Paget.

Os bisfosfonatos têm uma molécula formada por um carbono central em que estão ligados dois radicais fosfato mais um radical $\mathrm{R}_{1}$ (o ideal é que seja uma hidroxila) e um radical $\mathrm{R}_{2}$ (o ideal é que tenha uma cadeia cíclica e um átomo de nitrogênio neste radical). Dependendo da formação espacial desta molécula, ela tem mais ou menos capacidade de adsorção à molécula de hidroxia- 
patita. Sabe-se que a cadeia P-C-P com uma hidroxila em cada um destes átomos é a melhor formação para a adsorção do bisfosfonato à hidroxiapatita.

Esta adsorção é importante porque o osteoclasto, ao reabsorver o tecido ósseo, absorve também o bisfosfonato. Dentro do citoplasma do fagócito os bisfosfonatos aminados (aqueles que têm nitrogênio no radical $\mathrm{R}_{2}$ ) atuam na cadeia do mevalonato inibindo uma enzima, a farnesilpirofosfatosintase (FPPS). Esta enzima promove a tranformação do geranilpirofosfato em geranilgeranilpirofosfato e farnesilpirofosfato. Estes metabolitos promovem a prenilação das pequenas proteínas essenciais para a função da borda em escova e para a sobrevivência do osteoclasto. Assim, interrompendo a cadeia do mevalonato inibe-se a função osteoabsortiva do osteoclasto.

Os bisfosfonatos utilizáveis na terapêutica da OP diferenciam-se pela capacidade de adsorção ao cristal da hidroxiapatita e à potência com que inibem a função dos osteoclastos.

Os bisfosfonatos registrados no Brasil para o tratamento da osteoporose são: o alendronato de sódio, o pamidronato de sódio, o risedronato de sódio, o ibandronato de sódio e o ácido zoledrônico. Se considerarmos a potência antirreabsortiva comparando ao etidronato (como 1), teremos o alendronato como 1.000 vezes, o risedronato como 5.000 vezes, e o ibandronato e o ácido zoledrônico como 10.000 vezes. Quanto à capacidade de adsorção, a constante de afinidade de adsorção do etidronato é 1,2; do risedronato é 2,2; do ibandronato é 2,3; do ibandronato é 2,9; e do ácido zoledrônico é 3,4 ${ }^{(21)}$.

Os bisfosfonatos de uso oral têm baixa solubilidade e, por isso, devem ser administrados em jejum, com um copo de água pura (não é recomendada água mineral). O paciente deve ser mantido em jejum por mais meia hora. Como são agressivos para a mucosa esofágica o paciente não deve deitar-se na primeira meia hora, para aguardar o esvaziamento gástrico e evitar o refluxo esofágico.

Apenas 1\% é absorvido (0,6\% para o ibandronato). Destes, 51\% são eliminados por via renal, sem metabolização e 49\% são adsorvidos à hidroxiapatita, principalmente no osso novo. Quando liberados na corrente sanguínea, pela morte do osteoclasto ou por "desadsorção”, são novamente adsorvidos à hidroxiapatita. Alguns, como o risedronato, têm maior "desadsorção", o que explica sua melhor distribuição por todo o tecido ósseo e seu efeito multissítio.

Provavelmente estas diferenças já conhecidas, e outras ainda não conhecidas, fazem a diferença de ação antifratura dos vários bisfosfonatos. Semelhantes no modo de ação e diferentes na potência da ação, os bisfosfonatos atuam diferentemente na remissão da osteoporose densitométrica e na diminuição da prevalência de fraturas.

Aparentemente, a maior qualidade do alendronato é a experiência clínica acumulada. Como foi a primeira droga eficaz contra a osteoporose é usada há mais tempo e por um número maior de pessoas. Seu maior problema são os similares, não testados clinicamente, porém muito receitados, em substituição do sal original. Outro problema é a suspeita atual de provocar fraturas por forte inibição da remodelação óssea (frozen bone) quando usado por longo tempo. Foi testado na dose de 10mg diários, por via oral. Um estudo ponte mostrou que a dose de 70mg por semana também é eficiente como inibidor da incidência de FxOPs. Recentemente foi lançado com $70 \mathrm{mg}$ e $2.800 \mathrm{UI}$ de vitamina $\mathrm{D}_{3}$ para uso semanal e está para ser lançada a apresentação com 5.600UI, também para uso semanal.

O risedronato é o segundo mais usado em tempo de uso e tamanho da população usuária. Sua maior qualidade é a comprovada rapidez de ação e a eficácia antifratura multissítio com destaque para as fraturas de quadril, demonstrada em estudo clínico específico, o estudo Hip $^{(22)}$. Foi testado e lançado originalmente na dose de 5mg diários para uso oral. Um estudo ponte demonstrou sua eficácia com o uso semanal de 35mg e, agora, um novo estudo ponte demonstra sua validade para o uso mensal de $150 \mathrm{mg}^{(23)}$.

A maior qualidade do ibandronato é sua formulação com 150mg, para uso oral mensal. Já foi demonstrado que os bisfosfonatos orais podem ser administrados em doses maiores e com intervalos maiores, mantendo seu efeito na avaliação densitométrica. Originalmente foi lançado com 2,5mg para uso oral diário.

O ácido zoledrônico difere dos demais citados por ser de uso endovenoso, em dose anual. Também tem um estudo específico em pacientes com fratura do quadril, o estudo Horizon RFT ${ }^{(24)}$, no qual houve menor incidência de fraturas recorrentes no grupo droga ativa e o grupo tratado teve maior sobrevida que o grupo placebo. Por esta razão e pela vantagem de poder ser usado em paciente acamado, é muito indicado para uso em pacientes recém-operados de fraturas do fêmur proximal. Tem também a vantagem da aderência ao tratamento em função da posologia anual. Atualmente é registrado apenas para tratamento, mas o fabricante está aguardando liberação para uso também em prevenção da osteoporose. 
Os porcentuais de diminuição da incidência de fraturas e da remissão do quadro densitométrico dos vários bisfosfonatos, e também das demais terapêuticas, não são comparáveis, pois as populações estudadas, nos vários estudos disponíveis, foram muito diferentes entre si. Os estudos comparativos head to head (droga x droga) existentes ainda não são suficientes para se estabelecer uma grande diferença de umas sobre as outras ${ }^{(25)}$.

\section{Osteoprotegerina}

A osteoprotegerina é um produto que está chegando agora ao mercado, precedido por alguns anos de pesquisa. Atua inibindo o RANK, impedindo que ele se ligue ao osteoclasto para estimulá-lo a se reproduzir e para ativar a borda em escova do mesmo.

\section{Ranelato de estrôncio}

O ranelato de estrôncio é um produto para tratamento para osteoporose que apresenta as duas ações: é antirreabsortivo e ao mesmo tempo é pró-formador ${ }^{(26)}$.

O ranelato de estrôncio é um sal de ácido ranélico com dois átomos de estrôncio em cada molécula. É absorvido no intestino e a vitamina D não tem efeito nessa absorção. O ácido ranélico não é metabolizado, tem baixa ligação às proteínas plasmáticas, não se acumula no organismo humano e é rapidamente eliminado por via renal deixando os dois átomos de estrôncio livres para serem adsorvidos à hidroxiapatita (pequenas quantidades substituem os átomos de cálcio na composição do cristal) ${ }^{(27)}$.

A biodisponibilidade do estrôncio, administrado como 2,632g de ranelato de estrôncio hidratado (2g de anidro), é de $27 \%{ }^{(28)}$. O estrôncio atinge a concentração sérica máxima em três a cinco horas, tem meia vida de 62 horas e a parte não adsorvida à hidroxiapatita é excretada por via renal (57\%) e intestinal. O estrôncio não se liga a proteínas plasmáticas, não é metabolizado e não inibe o sistema citocromoP450. Atinge um ponto de equilíbrio em duas semanas e a meia vida é de 10 semanas.

O estrôncio é um elemento químico muito semelhante ao cálcio e ao magnésio. Tem valência +2 (como o cálcio e o magnésio), tem 38 elétrons distribuídos em quatro camadas (o cálcio tem 20 em três camadas), tem raio atômico de 215 (o cálcio tem 197), tem raio iônico de 116 (o cálcio tem 100). Estas semelhanças fazem o organismo confundi-los tanto na absorção intestinal quanto em sua participação no cristal de hidroxiapatita. A absorção depende do sal (o ácido ranélico foi desenvolvido para isto), da dose (neste caso, $2 \mathrm{~g}$ ), da presença do cálcio na dieta (administrar à noite, três horas após o jantar), da função renal e da espécie animal em estudo.
Como o estrôncio diminui a atividade da vitamina D3 hidroxilase, seu excesso pode levar o osso à osteomalácia. Nas pequenas doses diárias preconizadas estimula a calcificação normal do tecido osteoide.

Em cultura de tecido ósseo estimula a replicação do pré-osteoblasto, aumentando o número de osteoblastos e, portanto, aumenta a formação de osso. Estimula também a formação de colágeno.

Por outro lado, reduz a diferenciação dos osteoclastos e reduz sua atividade. Por isso inibe a reabsorção do osso. É então pró-formador e antirreabsortivo.

Os marcadores de formação óssea (fosfatase alcalina e pró-peptídeo C) aumentam e os de reabsorção óssea (C-telopeptídeo sérico e N-telopeptídeo urinário) diminuem (já no terceiro mês), confirmando sua dupla ação.

Em tecidos animais e biópsias humanas foi demonstrado que melhora a microarquitetura óssea ${ }^{(29,30)}$. Atua estimulando o volume trabecular, aumentando o número de trabéculas e a espessura das trabéculas. Não prejudica a qualidade óssea e a mineralização, por isso não deixa defeito mineral.

Além da formação de osso endostal, estimula a produção de osso periostal, o que melhora a macroarquitetura e a resistência do osso ${ }^{(30)}$.

Estudos mais recentes, utilizando tecnologia de ponta como a tomografia computadorizada quantitativa periférica de alta resolução (HR-pQCT), sugeriram que o ranelato de estrôncio age mais rapidamente e é mais eficaz na formação de osso novo cortical e trabecular do que o alendronato ${ }^{(31)}$, o que aponta para maior eficácia na prevenção de fraturas.

A presença do estrôncio no osso aumenta a absorção dos raios-X na densitometria, porém, um estudo comparativo recentemente publicado afirmou que a medida da DMO está relacionada a pelo menos 75\% da eficácia contra fraturas do ranelato de estrôncio, enquanto que para os bisfosfonatos esta estimativa está entre $4 \%$ e $28 \%{ }^{(32)}$.

Os estudos Soti e Tropos ${ }^{(33,34)}$ com duração até cinco anos comprovaram a eficácia do ranelato de estrôncio nos pacientes com osteoporose, dos seus estágios mais iniciais aos mais avançados, inclusive na população das pacientes com 80 ou mais anos de idade. Estes estudos comprovaram a redução do risco de fraturas vertebrais em $45 \%$ e redução das fraturas de quadril em $43 \%$ seja nos pacientes sem fraturas prévias (45\%), seja nos pacientes com fraturas (41\%).

\section{Escolha do tratamento}

O tratamento ideal é aquele que diminui a incidência de fraturas por melhorar a geometria do osso e sua micro- 
arquitetura. O tecido ósseo recém-formado deve ter boa qualidade celular e de matriz, mineralização normal com boa proporção entre osso mineralizado (resistente mecanicamente) e não mineralizado (flexível) e sem acúmulo de danos. O tratamento ideal deve ter taxa de remodelação positiva e efeito terapêutico rápido e duradouro. Este efeito deve ser facilmente detectável. Deve ser seguro.

Este tratamento ideal ainda não existe. Os vários tratamentos citados acima apresentam, cada um, algumas das características ideais e não apresentam outras. A escolha do tratamento, para cada paciente, depende das características do paciente, da gravidade da patologia e do conhecimento que o médico tenha do arsenal terapêutico como um todo e da medicação que vai prescrever em particular.

O problema do custo do tratamento estará sempre presente principalmente para evitar o abandono do tratamento. Compete aos médicos (e à sociedade) pressionar o poder público para permitir o uso do melhor tratamento que a sua consciência e seu conhecimento indicarem.

É preferível utilizar uma medicação que o médico tenha bom conhecimento das indicações, efeitos adversos, interação com outras drogas e contraindicações com outras patologias apresentadas pelo paciente.

As moléculas orgânicas apresentam isômeros espaciais que são quimicamente iguais e podem não sê-lo biologicamente. Similares e genéricos mais baratos podem ser até mais eficazes que os produtos de marca, mas não foram testados sob as rígidas exigências que as agências registradoras fazem. Em uma patologia de longo curso, que afeta pacientes idosos, não há tempo a perder com experiências com produtos mais baratos.

Algumas indicações são formais. O uso da teriparatida em pacientes com alto risco de uma fratura osteoporótica. O uso do risedronato quando se quer rapidez de ação, e ação multissítio, principalmente para prevenir fraturas do quadril. O uso do ácido zoledrônico quando se quer uma aderência ao tratamento de pelo menos um ano. O uso do ácido zoledrônico quando há indicação para o uso de bisfosfonatos e o paciente está acamado. O uso do ácido zoledrônico nos pacientes acamados em pós-operatório de fraturas do quadril. O uso da teriparatida e do ranelato de estrôncio quando se quer reativar o metabolismo ósseo aparentemente “congelado” pelo uso prolongado de alendronato.

É óbvio que nas osteoporoses secundárias é importante tratar a causa primária, mas em todas as osteoporoses, primárias e secundárias, o paciente pode ser beneficiado por qualquer um dos tratamentos acima.

\section{CONSIDERAÇÕES FINAIS}

\section{Avaliação da eficácia do tratamento}

A avaliação ideal seria a de testes mecânicos de resistência associados a exames anatomopatológicos ou histomorfométricos de ossos tratados. A diminuição da incidência de fraturas osteoporóticas vertebrais, não vertebrais e da extremidade proximal do fêmur também seria um bom avaliador. O problema é a praticidade destas avaliações. Assim, recorre-se à redução do risco relativo (RRR) de ocorrência de uma fratura osteoporótica, estabelecido por estaticistas, baseados em estudos clínicos e laboratoriais.

Há controvérsias sobre quanto cada droga reduz o risco relativo de cada fratura em cada população em particular. Não há controvérsias sobre a obrigação moral (e legal) do ortopedista, frente a um paciente com uma fratura osteoporótica, em tratá-lo ou encaminhá-lo para tratamento.

O melhor método de avaliação ainda é a densitometria. Resultados em prazo menor do que um ano são inconclusivos, por isso a primeira avaliação deve ser após um ano de tratamento, exceto na osteoporose induzida por glicocorticoides (que deve ser a cada seis meses). Quando a densitometria anual mostrar um ganho de massa óssea maior do que $2 \%$, esta avaliação pode ser bianual.

A microtomografia computadorizada quantitativa vertebral (mTCQV) nos dá uma imagem do trabeculado ósseo, donde se pode inferir a eficácia do tratamento. Não é usada na prática clinica diária porque é realizada por um aparelho ainda muito caro, mas é cada vez mais usada em pesquisas.

Os marcadores bioquímicos do rodízio ósseo são muito interessantes para pesquisas clínicas ou, na dúvida da eficácia do tratamento, em avaliações clínicas em prazo muito curto. Os marcadores séricos de formação e os marcadores de reabsorção óssea, em geral urinários, podem nos trazer informações já aos três meses de tratamento. Os marcadores de formação óssea mais estudados são: a fosfatase alcalina sérica total e sua fração óssea, a osteocalcina e os peptídeos procolágeno tipo I, carboxi e aminoterminal, séricos (própeptídeos C e N séricos). Os marcadores de reabsorção óssea mais estudados são: a hidroxiprolina urinária, os telopeptídeos N (NTx) e C (CTx) séricos e urinários, a piridinolina e a deoxipirinolina (DPD) urinárias, a fosfatase ácida tartarato resistente sérica e a calciúria. 


\section{REFERÊNCIAS}

1. Oliveira LG. Osteoporose. Guia para diagnóstico, prevenção e tratamento: Rio de Janeiro: Revinter; 2002.

2. NIH Consensus Development Panel on Osteoporosis Prevention, Diagnosis, and Therapy. Osteoporosis prevention, diagnosis, and therapy. JAMA. 2001;285(6):785-95.

3. World Health Organization - WHO. Prevention and management of osteoporosis. WHO Library Cataloguing-in-Publication Data; 2003.

4. Marshall D, Johnell O, Wedel H. Meta-analysis of how well measures of bone mineral density predict occurrence of osteoporotic fractures. BMJ. 1996;312(7041):1254-9.

5. Leslie WD, Metge C, Salamon EA, Yuen CK. Bone mineral density testing in healthy postmenopausal women. The role of clinical risk factor assessment in determining fracture risk. J Clin Densitom. 2002 Summer;5(2):117-30.

6. Siris ES, Brenneman SK, Barrett-Connor E, Miller PD, Sajjan S, Berger ML, et al. The effect of age and bone mineral density on the absolute, excess, and relative risk of fracture in postmenopausal women aged 50-99: results from the National Osteoporosis Risk Assessment (NORA). Osteoporos Int. 2006;17(4):565-74.

7. Kanis JA, Black D, Cooper C, Dargent P, Dawson-Hughes B, De Laet C, et al. A new approach to the development of assessment guidelines for osteoporosis. Osteoporos Int. 2002;13(7):527-36.

8. Kanis JA, Johnell O, Oden A, Dawson A, De Laet C, Jonsson B. Ten year probabilities of osteoporotic fractures according to BMD and diagnostic thresholds. Osteoporos Int. 2001;12(12):989-95.

9. Watts NB, Cooper C, Lindsay R, Eastell R, Manhart MD, Barton IP, et al. Relationship between changes in bone mineral density and vertebral fracture risk associated with risedronate: greater increases in bone mineral density do not relate to greater decreases in fracture risk. J Clin Densitom. 2004;7(3):255-61.

10. Gusmão CVB, Belangero, WD. Como a célula óssea reconhece o estímulo mecânico. Rev Bras Ortop. 2009;44(4):299-306.

11. Forsén L, Bjørndal A, Bjartveit K, Edna TH, Holmen J, Jessen V, et al. Interaction between current smoking, leanness, and physical inactivity in the prediction of hip fracture. J Bone Miner Res. 1994;9(11):1671-8.

12. Johnson LE. Deficiência, dependência e toxicidade das vitaminas. In: Manual Merck. $18^{\mathrm{a}}$ edição. São Paulo: Roca; 2006.

13. Hollis BW. Assessment of vitamin D status and definition of a normal circulating range of 25-hydroxyvitamin D. Curr Opin Endocrinol Diabetes Obes. 2008;15(6):489-94.

14. Dawson-Hughes B, Harris SS, Krall EA, Dallal GE. Effect of calcium and vitamin $D$ supplementation on bone density in men and women 65 years of age or older. N Engl J Med. 1997;337(10):670-6.

15. Jiang Y, Zhao JJ, Mitlak BH, Wang O, Genant HK, Eriksen EF. Recombinant human parathyroid hormone (1-34) [teriparatide] improves both cortical and cancellous bone structure. J Bone Miner Res. 2003;18(11):1932-41.

16. Uusi-Rasi K, Sievänen H, Pasanen M, Oja P, Vuori I. Associations of calcium intake and physical activity with bone density and size in premenopausal and postmenopausal women: a peripheral quantitative computed tomography study. J Bone Miner Res. 2002;17(3):544-52.

17. Miller PD, Bilezikian JP, Deal C, Harris ST, Ci RP. Clinical use of teriparatide in the real world: initial insights. Endocr Pract. 2004;10(2):139-48.
18. Lindsay R, Scheele WH, Neer R, Pohl G, Adami S, Mautalen C, et al. Sustained vertebral fracture risk reduction after withdrawal of teriparatide in postmenopausal women with osteoporosis. Arch Intern Med. 2004;164(18):2024-30.

19. Ettinger B, San Martin J, Crans G, Pavo I. Differential effects of teriparatide on BMD after treatment with raloxifene or alendronate. J Bone Miner Res. 2004;19(5):745-51.

20. Miller PD, Delmas PD, Lindsay R, Watts NB, Luckey M, Adachi J, et al. Early responsiveness of women with osteoporosis to teriparatide after therapy with alendronate or risedronate. J Clin Endocrinol Metab. 2008;93(10):3785-93.

21. Nancollas GH, Tang R, Phipps RJ, Henneman Z, Gulde S, Wu W, et al. Novel insights into actions of bisphosphonates on bone: differences in interactions with hydroxyapatite. Bone. 2006;38(5):617-27.

22. McClung MR, Geusens P, Miller PD, Zippel H, Bensen WG, Roux C, et al. Effect of risedronate on the risk of hip fracture in elderly women. Hip Intervention Program Study Group. N Engl J Med. 2001;344(5):333-40.

23. Delmas PD, McClung MR, Zanchetta JR, Racewicz A, Roux C, Benhamou CL, et al. Efficacy and safety of risedronate $150 \mathrm{mg}$ once a month in the treatment of postmenopausal osteoporosis. Bone. 2008;42(1):36-42.

24. Lyles KW, Colón-Emeric CS, Magaziner JS, Adachi JD, Pieper CF, Mautalen $\mathrm{C}$, et al. Zoledronic Acid in Reducing Clinical Fracture and Mortality after Hip Fracture. N Engl J Med. 2007;357:nihpa40967.

25. Silverman SL, Watts NB, Delmas PD, Lange JL, Lindsay R. Effectiveness of bisphosphonates on nonvertebral and hip fractures in the first year of therapy: the risedronate and alendronate (REAL) cohort study. Osteoporos Int. 2007;18(1):25-34.

26. Marie PJ, Ammann P, Boivin G, Rey C. Mechanisms of action and therapeutic potential of strontium in bone. Calcif Tissue Int. 2001;69(3):121-9

27. Bonnelye E, Chabadel A, Saltel F, Jurdic P. Dual effect of strontium ranelate: stimulation of osteoblast differentiation and inhibition of osteoclast formation and resorption in vitro. Bone. 2008;42(1):129-38.

28. Marie PJ. Optimizing bone metabolism in osteoporosis: insight into the pharmacologic profile of strontium ranelate. Osteoporos Int. 2003;14 (Suppl 3):S9-12.

29. Farlay D, Boivin G, Panczer G, Lalande A, Meunier PJ. Long-term strontium ranelate administration in monkeys preserves characteristics of bone mineral crystals and degree of mineralization of bone. J Bone Miner Res. 2005;20(9):1569-78.

30. Arlot ME, Jiang Y, Genant HK, Zhao J, Burt-Pichat B, Roux JP, et al. Histomorphometric and microCT analysis of bone biopsies from postmenopausal osteoporotic women treated with strontium ranelate. J Bone Miner Res. 2008;23(2):215-22.

31. Rizzoli R. Beneficial effects of strontium ranelate compared to alendronate on bone micro structure: a 2 year study. Osteoporosis Int. 2010; 21:(Suppl 1): S25-S388.

32. Kendler DL, Adachi JD, Josse RG, Slosman DO. Monitoring strontium ranelate therapy in patients with osteoporosis. Osteoporos Int. 2009;20(7):1101-6.

33. Meunier PJ, Roux C, Seeman E, Ortolani S, Badurski JE, Spector TD, et al. The effects of strontium ranelate on the risk of vertebral fracture in women with postmenopausal osteoporosis. N Engl J Med. 2004;350(5):459-68.

34. Reginster JY, Sawicki A, Roces-Varela A. Strontium Ranelate: Long-term efficacy over 8 years in Post menopausal osteoporotic women. Arthritis Rheum. 2008;58:S941. 